\title{
MODELING DRUG RELEASE FROM DYNAMICALLY SWELLING POLY(HYDROXYETHYL METHACRYLATE-CO-METHACRYLIC ACID) HYDROGELS
}

\author{
Jim H. Kou*, David Fleisher and Gordon L. Amidon \\ College of Pharmacy, The University of Michigan, Ann Arbor, Michigan 48109 (U.S.A.)
}

(Received May 26, 1989; accepted in revised form October 2, 1989)

Keywords: drug release; $\mathrm{pH}$-dependent swelling; diffusion; free volume; hydrogels

Drug release from a swelling hydrogel matrix is a complicated process where diffusion of drug molecules is coupled to the swelling kinetics. The swelling influences the diffusional flux of drug molecules by increasing the diffusion coefficient and diffusional pathlength. Release of phenylpropanolamine from a poly (hydroxyethyl methacrylate-co-methacrylic acid) hydrogel, initially at low $\mathrm{pH}$, is highly influenced by swelling in a neutral $\mathrm{pH}$ medium. Swelling in these ionizing gels is a very long process, even in the absence of a glassy core, indicating that the swelling is not a simple Fickian process. In this paper, a novel approach is introduced to model the phenylpropanolamine release from these swelling gels. Through a free volume relationship, the experimentally determined swelling kinetics are coupled to a diffusion mechanism for the drug molecules. This model was shown to give an accurate prediction of phenylpropanolamine release from poly(hydroxyethyl methacrylate-co-methacrylic acid) gels.

\section{INTRODUCTION}

Hydrogels are essentially hydrophilic polymers capable of taking up a significant amount of water without themselves dissolving. Poly(hydroxyethyl methacrylate-co-methacrylic acid) [poly(HEMA-co-MA)] gels, initially swollen at low $\mathrm{pH}$, were shown to swell extensively in a neutral pH medium [1]. These gels contain ionizable carboxylic side chains which are responsible for the extent of swelling. A typical gel sample with $30 \%$ of methacrylic acid and $0.5 \%$ of crosslinker can absorb up to $84 \%$ of water. These ionizing gels often swell

*To whom correspondence should be addressed. Present address: Pharmaceutical R\&D, Genentech, Inc., 460 Point San Bruno Boulevard, South San Francisco, CA 94080, U.S.A. very slowly, and the mechanism is yet to be elucidated. For poly (acrylamide-co-acrylic acid) gels, it was suggested that the rate of swelling is controlled by the rate of ion exchange as well as the boundary layer [2]. Therefore, unlike swelling of initially dry neutral hydrogels, swelling kinetics of these ionizable gels can significantly deviate from Fickian sorption.

Drug release from these swelling gels is consequently a complicated phenomenon, where the diffusion of drug molecules is superimposed on the medium swelling. The swelling will alter the properties of the matrix, thus changing the diffusional characteristics of the drug molecules. It was determined that the diffusion coefficient of phenylpropanolamine (PPA) in a poly (HEMA-co-MA) matrix increased 52-fold when the hydration level increased from $30 \%$ at $\mathrm{pH} 3$ to $88 \%$ at $\mathrm{pH} 7$ [1]. Consequently, the 
release kinetics of loaded drug depend highly on the swelling kinetics. Transport analysis on the drug release from $\mathrm{pH}$-sensitive gels have been published for both anionic and cationic hydrogels $[3,4]$. Starting from the dry matrix, it was found that the swelling extends the linear portion of the release curve beyond $80 \%$ of the total release, indicating a swelling induced anomalous release. Several mathematical models were developed previously to describe the drug release from swellable systems [5-10]. These models used Fick's equation as the governing equation for drug transport. Matrix swelling was also treated explicitly in most of these models by assuming a Fickian sorption. The effect of swelling on drug release was then coupled through a diffusion coefficient with penetrant concentration dependence. Since we have argued against the diffusion mechanism for swelling in poly (HEMA-co-MA) gels, it is clear that an alternative approach should be taken to model the drug release from these gels. More recently, release from a gel matrix undergoing anomalous swelling was treated theoretically, incorporating explicitly a glassy to rubbery transition front [11]. However, our current work is focused on the release from a gel matrix swelling from a pre-swollen state without the influence of the glassy core.

The purpose of this paper is to introduce a general approach to predict the drug release from a hydrogel matrix undergoing $\mathrm{pH}$-induced swelling with unknown mechanism. The rationale of the approach is described as follows. The diffusional flux, $J$, of drug through a polymer can be written as:

$J=\frac{\Delta C}{\Omega}$

where $\Delta C$ is the concentration gradient and $\Omega$ is the resistance, which is defined as:

$\Omega=\frac{L}{D}$

where $L$ and $D$ are the diffusion pathlength and the drug diffusion coefficient, respectively. When the polymer is undergoing swelling, $\Omega$ becomes time dependent. For highly hydrophilic systems, both $L$ and $D$ will increase with time as a result of swelling. Given the complex nature of swelling, the time dependence of $L$ and $D$ will be determined experimentally. Drug release rate can then be predicted via a diffusional model using these measured variables.

\section{THEORY}

The non-steady swelling release problem is formulated in cylindrical geometry because of the flexibility to include a range of geometries from a long rod to a thin slab. The mass transfer equation for the two-dimensional diffusion problem in cylindrical coordinates can be written as:

$\frac{\partial C_{2}}{\partial t}=\frac{1 \partial}{r \partial r}\left(r D_{2} \frac{\partial C_{2}}{\partial r}\right)+\frac{\partial}{\partial z}\left(D_{2} \frac{\partial C_{2}}{\partial z}\right)$

where $C_{2}$ is concentration and $D_{2}$ is the penetrant concentration dependent diffusion coefficient of drug molecules. It has been shown that the solute diffusion coefficient in hydrophilic membranes follows the free volume theory of Yasuda et al. [12], which is expressed as:

$D_{2}=D_{2,0} \mathrm{e}^{-k_{\mathrm{f}}(1 / H-1)}$

where $H, D_{2,0}$ and $k_{\mathrm{f}}$ are, respectively, matrix hydration, solute diffusivity in water and Yasuda's free volume parameter. Matrix hydration is defined as the fractional water content. This correlation was established previously with PPA diffusion in poly (HEMA-co-MA) gels [1]. The current approach is to determine $H$ experimentally where the measured quantity is a spatially averaged value. Therefore, $H$ is a function only of time, and the penetrant concentration distribution within the hydrogel network is thus not considered. Consequently, the diffusion coefficient is also a spatially averaged quantity.

The boundary and initial conditions are: 
$C=K C_{2, \mathrm{~b}}(t), r=R(t), \quad 0 \leqslant z \leqslant Z(t), t>0(5)$

$\frac{\partial C_{2}}{\partial r}=0, \quad r=0, \quad 0 \leqslant z \leqslant Z(t), t>0(6)$

$C=K C_{2, \mathrm{~b}}(t), z=Z(t), \quad 0 \leqslant r \leqslant R(t), t>0(7)$

$C=K C_{2, \mathrm{~b}}(t), z=0, \quad 0 \leqslant r \leqslant R(t), t>0(8)$

$C=C_{2,0}, \quad 0 \leqslant r \leqslant R(t), 0 \leqslant z \leqslant Z(t), t=0(9)$

where $R, Z, K, C_{2, \mathrm{~b}}$ and $C_{2,0}$ denote cylinder radius, cylinder length, partition coefficient, concentration in bulk fluid and loading concentration, respectively. $R(t)$ and $Z(t)$ define the time-dependent boundaries during swelling, and $C_{2, \mathrm{~b}}$ allows for the solute concentration buildup in the bulk, so that the method is not limited only to sink conditions. At the center axis of the cylinder, the zero flux condition is necessary for symmetry.

Due to the complexity, the model was solved by a numerical finite difference method. The boundary conditions can be simplified by the following variable transformations:

$$
\begin{aligned}
\hat{r} & =\frac{r}{R(t)} \\
\hat{z} & =\frac{z}{Z(t)} \\
\tau & =\frac{D_{2,0} t}{R(0)^{2}} \\
\theta & =\frac{C_{2}}{C_{2,0}}
\end{aligned}
$$

where $R(0)$ is the initial cylinder radius. The transformed equation becomes:

$$
\frac{\partial \theta}{\partial \tau}=\frac{D_{2}^{*}}{R^{* 2}}\left(\frac{1 \partial \theta}{\hat{r} \partial \hat{r}}+\frac{\partial^{2} \theta}{\partial \hat{r}^{2}}\right)+\frac{D_{2}^{*}}{\left(Z^{*} \gamma\right)^{2}} \frac{\partial^{2} \theta}{\partial \hat{z}^{2}}
$$

where the various dimensionless parameters are defined by:

$$
\begin{aligned}
& R^{*}=\frac{R(\tau)}{R(0)} \\
& Z^{*}=\frac{Z(\tau)}{Z(0)}
\end{aligned}
$$

$$
\begin{aligned}
D_{2}^{*} & =\frac{D_{2}(H)}{D_{2,0}}=\mathrm{e}^{-k f(1 / H-1)} \\
\gamma & =\frac{Z(0)}{R(0)}
\end{aligned}
$$

The transformed boundary and initial conditions are:

$\theta=K \frac{C_{2, \mathrm{~b}}}{C_{2,0}}, \quad \hat{r}=1, \quad 0 \leqslant \hat{z} \leqslant 1, \quad \tau>0$

$\frac{\partial \theta}{\partial \hat{r}}=0, \quad \hat{r}=0, \quad 0 \leqslant \hat{z} \leqslant 1, \quad \tau>0$

$\theta=K \frac{C_{2, \mathrm{~b}}}{C_{2,0}}, \quad \hat{z}=1, \quad 0 \leqslant \hat{r} \leqslant 1, \quad \tau>0$

$\theta=\frac{C_{2, \mathrm{~b}}}{C_{2,0}}, \quad \hat{z}=0, \quad 0 \leqslant \hat{r} \leqslant 1, \quad \tau>0$

$\theta=1, \quad 0 \leqslant \hat{r} \leqslant 1, \quad 0 \leqslant \hat{z} \leqslant 1, \quad \tau=0$

Significance of this transformation is to clear the time dependence from the boundary conditions and scale them into the partial differential equation. The transformed problem thus becomes a fixed boundary one. The ratio of $C_{2, \mathrm{~b}} /$ $C_{2,0}$ can be expressed as $\left[M_{2}(\tau) / M_{2, \infty}\right]\left(V_{\mathrm{g}} / V_{\mathrm{b}}\right)$, where $M_{2}(\tau) / M_{2, \infty}$ is the fractional drug released at time $\tau$ and $V_{\mathrm{g}} / V_{\mathrm{b}}$ is the volume ratio of gel and bulk phases. The boundary concentration in eqns. (19), (21) and (22) may then be expressed as:

$\theta=K\left[\frac{M_{2}(\tau)}{M_{2, \infty}}\right]\left(\frac{V_{\mathrm{g}}}{V_{\mathrm{b}}}\right)$

where the fractional drug release at time $\tau$ can be calculated by:

$\frac{M_{2}(\tau)}{M_{2, \infty}}=1-2 R^{* 2} Z^{*} \int_{0}^{1} \int_{0}^{1} \theta \hat{r} \mathrm{~d} \hat{r} \mathrm{~d} \hat{z}$

This also allows us to follow the boundary conditions with time according to eqn. (24). Equation (14) was solved along with boundary conditions (19)-(23) via an explicit finite dif- 
ference scheme. The scheme was shown to be consistent and the stability condition was established as:

$$
\Delta \tau=\frac{(\Delta \hat{s})^{2}}{2 D^{*}\left(\frac{2}{R^{*}}+\frac{1}{\left(Z^{*} \gamma\right)^{2}}\right)}
$$

where $\Delta \hat{s}$ and $\Delta \tau$ are the dimensionless spatial and time step sizes. Here the spatial step sizes in both $\hat{r}$ and $\hat{z}$ are taken to be the same, i.e. equal to $\Delta \hat{s}$. The soluble integral in eqn. (25) was evaluated by a two-dimensional Simpson formula [13].

\section{EXPERIMENTAL METHODS}

\section{Hydrogel synthesis}

The monomer reagents, hydroxyethyl methacrylate (HEMA), methacrylic acid (MA) and tetraethylene glycol dimethacrylate (TEGDMA), were obtained from Polyscience Inc. (Warrington, PA). The purification procedure for HEMA and MA monomers was described previously [1]. The hydrogel cylinders were fabricated in polyethylene tubing ( $3 \mathrm{~mm}$ I.D.) via bulk polymerization. The methacrylic acid concentrations used were 10,20 and $30 \%$ (w/ $w$ ), and the TEGDMA concentration was fixed at the level of $0.2 \%(\mathrm{w} / \mathrm{w})$. The monomeric mixture was initiated by $1 \% 2,2^{\prime}$-azobisisobutyronitrile (AIBN) and injected into straight polyethylene tubing. The two ends were than sealed and the entire enclosure was maintained in a $60^{\circ} \mathrm{C}$ water bath for $12 \mathrm{~h}$. The resulting polymer cylinder was cut into $3 \mathrm{~cm}$ segments and washed in portions of freshly distilled water for a period of at least one week before further use.

\section{Release of phenylpropanolamine}

Phenylpropanolamine (PPA), obtained from Sigma Chemical Co. (St. I ouis, MO), was cho- sen as the model compound to study because of its good water solubility and stability. For release experiments, drug loading was achieved by equilibrating gel cylinders in 5\% PPA solutions at $\mathrm{pH} 1$ and total ionic strength of $0.37 \mathrm{M}$. The loading period was estimated by $R^{2} / D_{1}$, where $R$ and $D$ are cylinder radius and PPA diffusivity in the gel respectively. Using the value of $0.15 \mathrm{~cm}$ for $R$ and $2.5 \times 10^{-8} \mathrm{~cm}^{2} / \mathrm{s}$ for $D$, the loading time would be approximately 11 days. The loaded gel was then removed, blotted, and transferred to an extracting solution. No drying was allowed during this transfer. The loaded polymer sample was placed horizontally on a glass slide engraved with a micrometer scale and anchored by two stainless steel wires. This arrangement was to allow the radius to be determined by photographic techniques. The extracting solution was a $\mathrm{pH} 7$ phosphate buffer with total ionic strength maintained at $0.37 \mathrm{M}$ by the buffer species. The volume of the extracting solution was $150 \mathrm{ml}$ and the solution was stirred at $120 \mathrm{rpm}$ to ensure a uniform bulk concentration. The stirring rate was shown to have no effect on the release kinetics over the range 120-390 rpm. Thus, the boundary layer effect was assumed to be insignificant in these experiments. The bulk PPA concentration and the radius, length and weight of the polymer cylinder were followed as a function of time throughout the entire release period. PPA samples were assayed by an ion pairing HPLC method.

\section{RESULTS AND DISCUSSION}

\section{Effect of $\gamma$ on drug release}

The parameter $\gamma$, defined in eqn. (18), is the initial aspect ratio of the cylinder. If $\gamma$ is much greater than 1 , the cylinder assumes the shape of a long rod, while if $\gamma$ is smaller than one, it becomes a thin disk. Figure 1 illustrates the effect of $\gamma$ on the release rate. The release rate is apparently increased when the polymer cylin- 


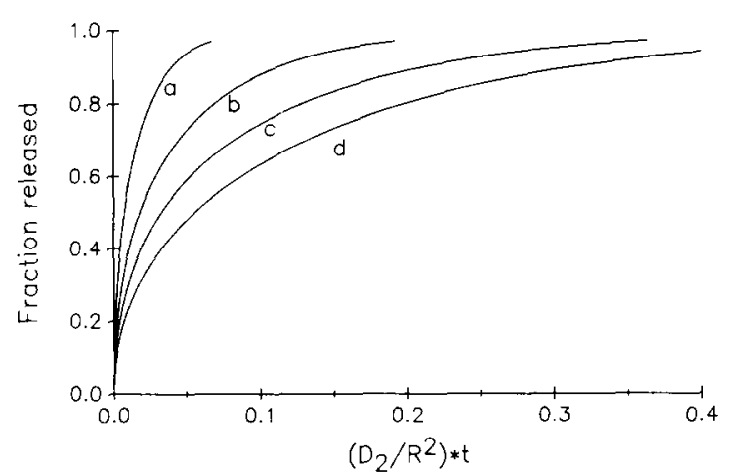

Fig. 1. Effect of $\gamma$ on release rate. Labels $a, b, c$ and $d$ represent $\gamma$ values of $0.5,1,2$ and 10 respectively $\left(R^{*}=Z^{*}=1\right.$; $\left.k_{\mathrm{f}}=0 ; K=0\right)$.

der is shortened from a long rod to a thin disk by decreasing $\gamma$. This trend is the result of a shift in the relative importance of the axial and radial resistances in determining the release rate. From Fig. 1, it is clear that the release rate is most sensitive to the $\gamma$ value around unity. Within this range, the cylinder is in the shape of a tablet, so one can effectively manipulate the aspect ratio of a tablet alone to control the release rate from the matrix. This approach may be useful in practical dosage form design for controlled release.

\section{Effect of $K\left(V_{g} / V_{b}\right)$ on release rate}

The advantage of using the boundary conditions of eqns. (19), (21) and (22) is that one does not have to maintain a sink condition in the experimental setting. Since it is clear from eqn. (24) that the partition coefficient and volume ratio influence the transport rate in the same way, i.e. by modifying the concentration at the boundary, only the effect of the combined parameter, $K\left(V_{\mathrm{g}} / V_{\mathrm{b}}\right)$, needs to be analyzed. Figure 2 is the plot of fraction released versus time, demonstrating the effect of $K\left(V_{\mathrm{g}} / V_{\mathrm{b}}\right)$. The release declines and levels off prematurely as $K\left(V_{\mathrm{g}} / V_{\mathrm{b}}\right)$ is increased. The reason is clearly due to the diminished driving force for diffusion when $K\left(V_{\mathrm{g}} / V_{\mathrm{b}}\right)$ is large. Comparing curves $2 a$ and $2 b$, it can be seen that the error intro-

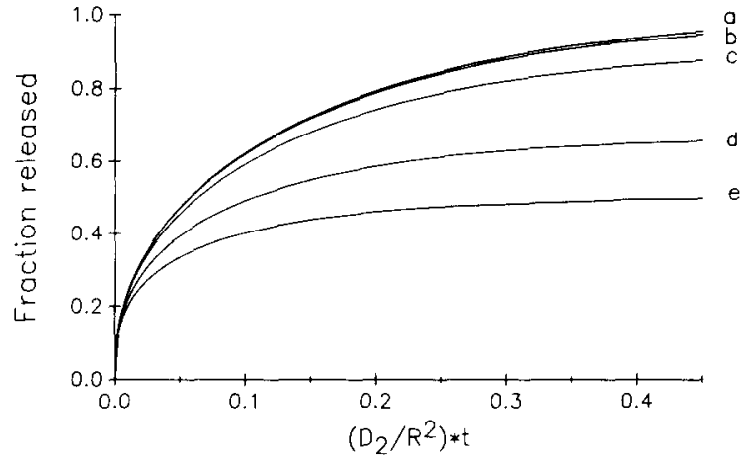

Fig. 2. Effect of $K\left(V_{\mathrm{g}} / V_{\mathrm{b}}\right)$ on release rate. Labels a, b, c, d and e represent, respectively, $K\left(V_{\mathrm{g}} / V_{\mathrm{b}}\right)$ values of $0,0.01$, $0.1,0.5$ and $1.0\left(R^{*}=Z^{*}=1 ; k_{\mathrm{f}}=0 ; \gamma=20\right)$.

duced in assuming a sink condition is only $0.85 \%$, determined at $\tau=0.4$, when the value of $K\left(V_{\mathrm{g}} / V_{\mathrm{b}}\right)$ is 0.01 .

\section{Effect of penetrant concentration gradient in a swelling gel matrix on release rate}

In the present model, swelling and drug release are coupled through a penetrant concentration dependent diffusion coefficient of the drug. This is shown in eqn. (4), where the penetrant concentration is expressed in terms of matrix hydration, $H$. Rigorously, one should treat $H$ as a function of time and spatial variables, assuming the swelling mechanism is known a priori. The present approach is to measure $H$ experimentally, giving a spatially averaged quantity which is a function only of time. To justify the current approach, it is necessary to compare the fraction released vs. time curves between the rigorous treatment, i.e. considering $H\left[C_{1}(x, t)\right]$, and the spatially averaged approach, i.e. using $H(t)$. In order to make the analysis, a mechanism for the penetrant swelling must be assumed. In this regard, a Fickian mechanism was chosen, since the solution to the mathematical problem is readily available. The details of the model are presented in the appendix, and the results are discussed below.

The rate determining parameters in the model are $k_{\mathrm{f}}$ and $D_{1} / D_{2,0}$, where $k_{\mathrm{f}}$ is the free 
volume parameter as defined in eqn. (4) and $D_{1} / D_{2,0}$ is the ratio of the penetrant diffusion coefficient in the gel matrix and the drug diffusion coefficient in the bulk penetrant. Both $k_{\mathrm{f}}$ and $D_{2,0}$ have been determined experimentally for the PPA/poly (HEMA-co-MA) system [1] and the values are 1.98 and $1.82 \times 10^{-6}$ $\mathrm{cm}^{2} / \mathrm{s}$ respectively. For $D_{1}$, one can use the water diffusivity measured in poly(hydroxyethyl methacrylate-co- $N$-vinylpyrrolidone) gels [14]. Taking an average matrix hydration of $0.50, D_{1}$ is estimated to be $2.08 \times 10^{-6} \mathrm{~cm}^{2} / \mathrm{s}$. Since $D_{2,0}$ and $D_{1}$ are comparable, a $D_{1} / D_{2,0}$ value of 1 will be used in the analysis. Figure 3 is the plot of fraction released vs. time comparing to sets of swelling limits assuming $k_{\mathrm{f}}=2$ and $D_{1} / D_{2,0}=1$. $H(0)$ and $H(\infty)$ are the initial and final matrix hydrations respectively. The result shows that the cumulative release kinetics are essentially

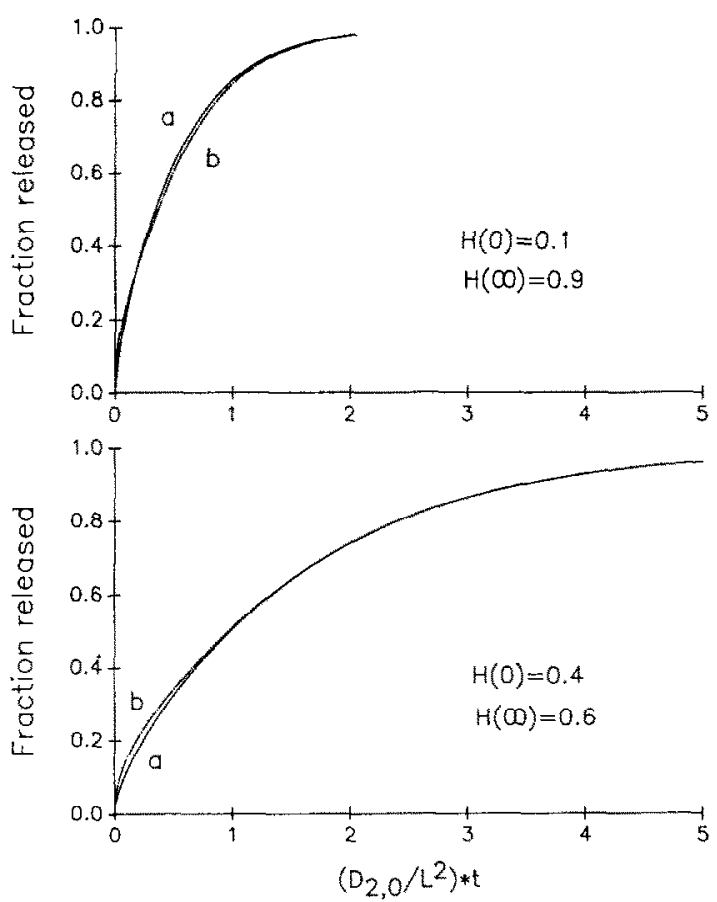

Fig. 3. Effect of penetrant concentration distribution on release rate with $k_{\mathrm{f}}=2$ and $D_{1} / D_{2,0}=1$. Curves (a) and (b) are computed based on eqns. (A7) and (A8), respectively. Top graph: $H(0)=0.1$ and $H(\infty)=0.9$. Bottom graph: $H(0)=0.4$ and $H(\infty)=0.6$. the same whether one uses $H\left[C_{1}(x, t)\right]$, curve $3(\mathrm{a})$, or the spatially averaged $H(t)$, curve $3(\mathrm{~b})$, for both sets of swelling limits. Clearly, this shows that the overall mass transfer resistance to the drug species in the gel matrix is the same, whether or not the penetrant concentration gradient is considered.

\section{Release dynamics}

The model predicts the drug release rate based on the experimentally determined swelling. Quantities needed for release simulation include $k_{\mathrm{f}}, D_{2,0} K, V_{\mathrm{g}} / V_{\mathrm{b}}, R^{*}(\tau), Z^{*}(\tau)$ and $H(\tau)$. The $k_{f}, D_{2,0}$ and $K$ values for the PPA/ poly (HEMA-co-MA) system have been determined independently and reported previously [1]. The values used here are $k_{\mathrm{f}}=1.98$ and $D_{2,0}=1.82 \times 10^{-6} \mathrm{~cm}^{2} / \mathrm{s}$. Since the initial gel volume is about $0.21 \mathrm{~cm}^{3}$ and extracting bulk volume is $150 \mathrm{~cm}^{3}, V_{\mathrm{g}} / V_{\mathrm{b}}$ is calculated to be 0.0014 . The largest $K$ value is 4.6 at $\mathrm{pH} 7$, and this yields a $K\left(V_{\mathrm{g}} / V_{\mathrm{b}}\right)$ value of 0.0064 , which indicates that the extracting fluid acts essentially as a sink for drug release. The time profiles of $R(t), Z(t)$ and $H(t)$ were determined experimentally. $R(t)$ and $Z(t)$ were dedimensionalized by their respective initial values to obtain the $R^{*}(t)$ and $Z^{*}(t)$ functions.

Figure 4 illustrates the experimental data and predicted release curve for a typical poly-

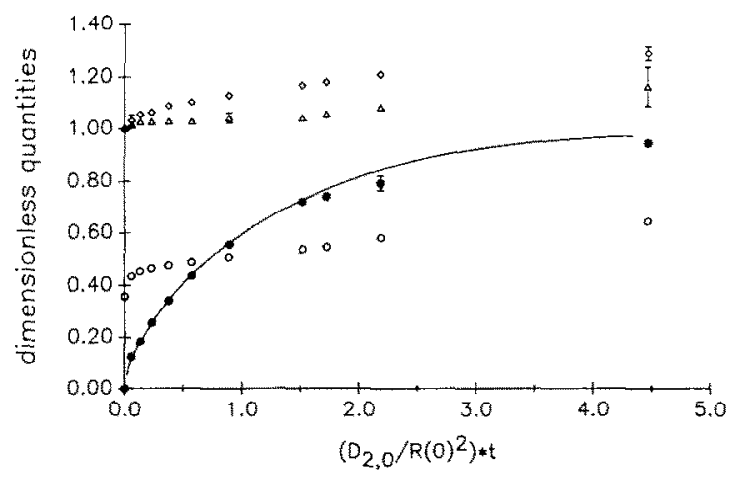

Fig. 4. Release of PPA from $20 \%$ MA poly (HEMA-co-MA) gels. $\odot, \triangle, \triangle$ and $\diamond$ are fraction released, $H, R^{*}$ and $Z^{*}$, respectively. Error bars indicate S.D. 
(HEMA-co-MA) gel consisting of $20 \%$ of methacrylic acid and $0.2 \%$ of the crosslinker TEGDMA. Also plotted in the graph are the experimental swelling data, i.e. $H, R^{*}$ and $Z^{*}$. The result shows that the model provides an excellent prediction of PPA release based on the swelling data. This is substantiated in Fig. 5, where the releases from the swelling and nonswelling matrices are compared. The top and bottom curves are the non-swelling releases at pH 7 and 1, respectively, and the middle is the same release curve as presented in Fig. 4 . It is apparent that these model predicted curves do describe the release adequately in both swelling and non-swelling cases.

The theoretical analysis in the previous section shows that using a spatially averaged $H(t)$ does not introduce a significant error to the release rate. This conclusion provides the basis for explaining the success of the model prediction. The matrix hydration $H$ for the $20 \% \mathrm{MA}$ gels increases from 0.36 to 0.62 during the drug release period. After an initial rapid phase, the swelling proceeds slowly to equilibrium. This equilibrium is reached at a much slower rate than the release equilibrium. The swelling matrix thus presents itself as an approximately uniform, albeit time dependent, medium to the diffusing PPA molecules.

Similar release experiments were conducted

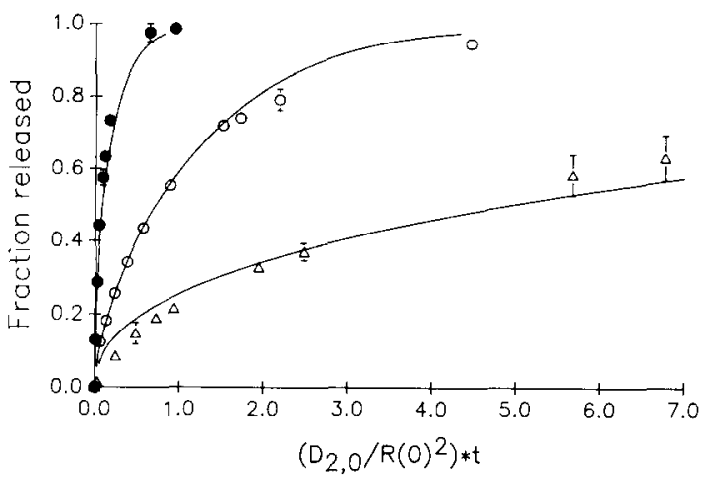

Fig. 5. Fractional PPA release from: non-swelling matrix at $\mathrm{pH} 1$ ( $\Delta$; loading at $\mathrm{pH} 1$ ), swelling matrix at $\mathrm{pH} 7(\mathrm{O}$; loading at $\mathrm{pH} 1)$, and non-swelling matrix at $\mathrm{pH} 7(\mathbf{O}$; loading at $\mathrm{pH} 7$ ).

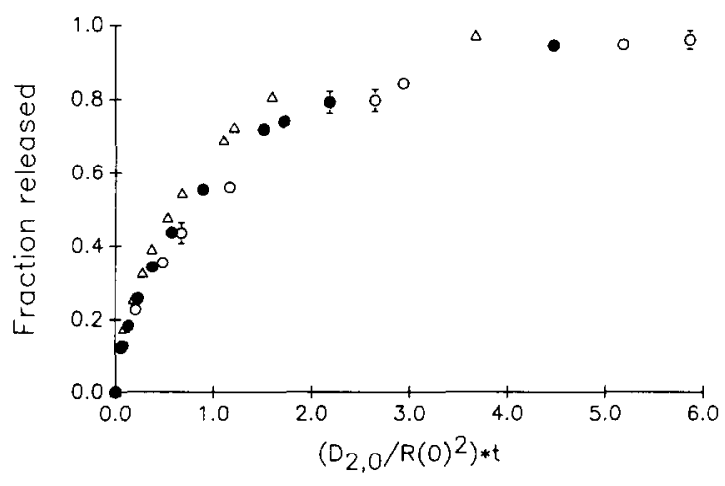

Fig. 6. Comparison of fraction released of PPA from $10 \%$ $(\triangle), 20 \%(\bigcirc)$, and $30 \%(O)$ MA poly (HEMA-co-MA) gels.

using poly (HEMA-co-MA) gels with $10 \%$ and $30 \%$ methacrylic acid content. Comparing the experimental release data from the three gels shows an interesting result, as illustrated in Fig. 6 . Gels with higher methacrylic acid content actually exhibit a slower PPA release rate. This result is somewhat unexpected because, according to free volume theory, drug release from matrices that swell more rapidly should be faster. These data can be explained by considering the swelling data in light of the dimensional changes. Figure 7 compares $H, R^{*}$ and $Z^{*}$ as measured during the release experiments. While the matrix hydration, $H$, increases more rapidly in gels with higher methacrylic acid content [Fig. 7(a)], the dimensional increase is also more significant [Figs. 7(b) and 7(c) ]. In general, diffusional resistance can be expressed by eqn. (2); therefore, the drug release rate is the result of a balance between $L$ and $D$. In fact, the radial resistance, $\Omega_{\hat{r}}$, in the gel can be calculated by,

$\Omega_{\hat{r}}=\frac{R^{*}}{D_{2}^{*}}$

Figure 8 illustrates the time profiles of computed $\Omega_{\hat{r}}$ for all three gels. It is clear that, initially, $\Omega_{\hat{r}}$ is substantially greater in gels of higher methacrylic acid content, thus resulting in a slower release rate. Although the resistances decrease rapidly with time and eventually cross, 

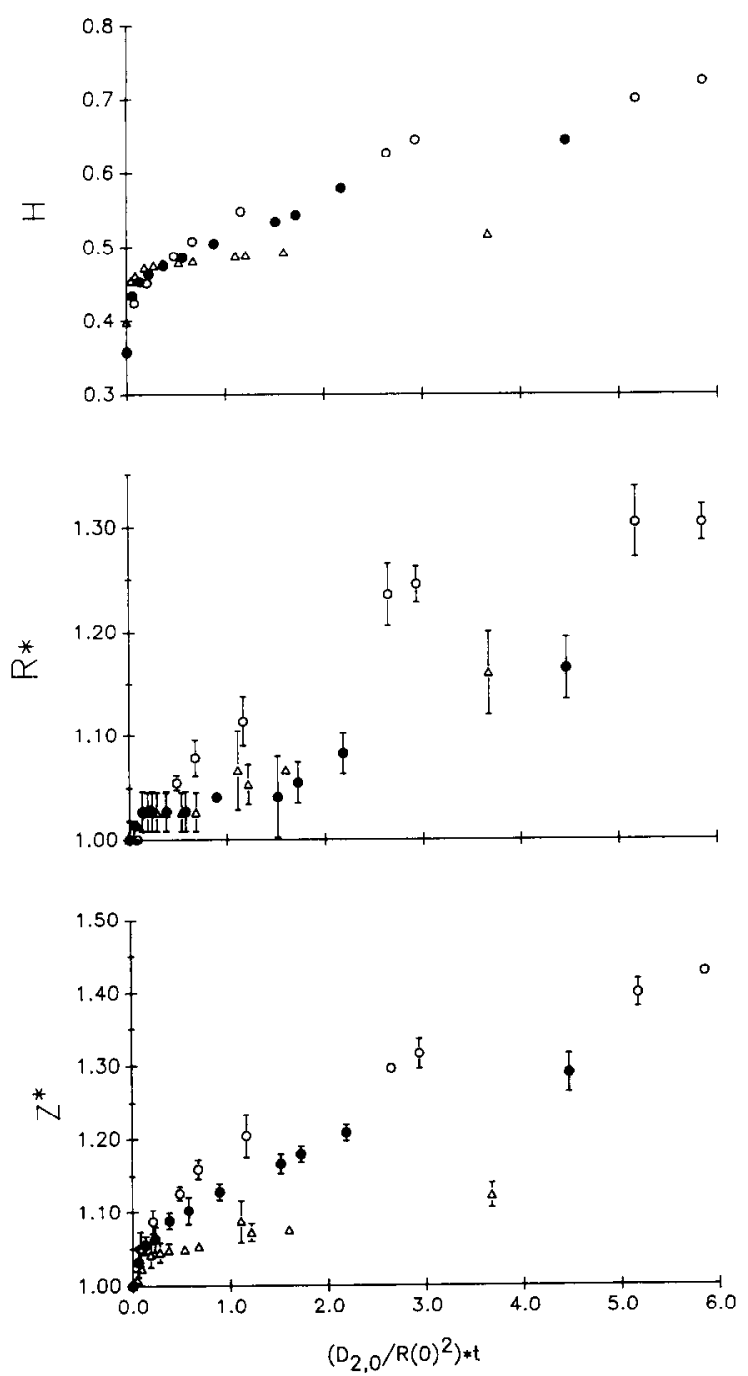

Fig. 7. Comparisons of experimental $H, R^{*}$ and $Z^{*}$ of $10 \%$ $(\triangle), 20 \%(\bigcirc)$ and $30 \%(O)$ MA poly (HEMA-co-MA) gels.

they level off in a fairly narrow range. There is no corresponding crossover observed in the release curves because, at this later stage of desorption, the driving force is considerably diminished and a small difference in resistance will not greatly modify the cumulative release behavior.

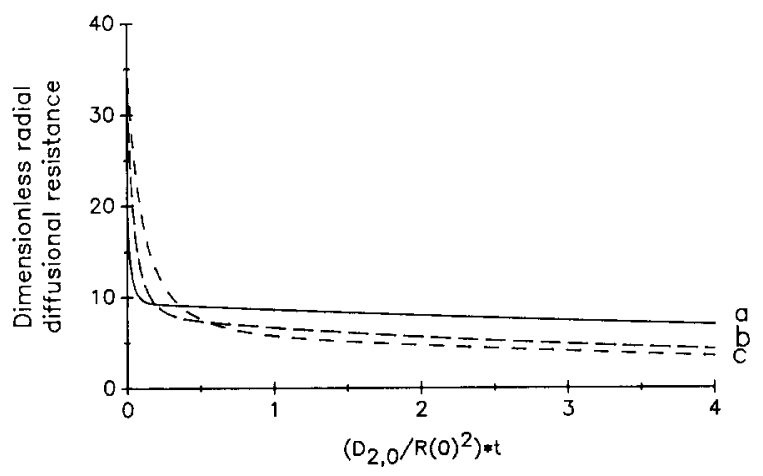

Fig. 8. Comparison of radial diffusional resistances, $\Omega_{\hat{r}}$, for PPA in $10 \%$ (a), $20 \%$ (b) and $30 \%$ (c) poly (HEMA-coMA) gels.

\section{CONCLUSIONS}

While the drug release from an ionizing and swelling gel is a complicated phenomenon, an approach to predict the release rate has been developed using experimental swelling data. With the measured quantities, such as matrix hydration and matrix dimensions, a diffusional model is solved to give the drug release profile. The success in applying the model to predict the release of phenylpropanolamine from swelling poly(HEMA-co-MA) gels indicates that the release kinetics are controlled by the matrix swelling. The apparent release curves from gels of various MA content were also adequately explained, based on the analysis of mass transfer resistance. The relative balance between the increase in diffusion coefficient and the increase in dimensions is found to determine the release rate from these gel matrices.

\section{ACKNOWLEDGEMENT}

The authors wish to thank the Smith Kline and Beckman Corporation for their financial support of this work. 


\section{REFERENCES}

1 J.H. Kou, G.L. Amidon and P.I. Lee, pH-Dependent swelling and solute diffusion characteristics of poly (hydroxyethyl methacrylate-co-methacrylic acid) hydrogels, Pharm. Res., 5 (9) (1988) 539-597.

2 S.H. Gehrke and E.L. Cussler, Mass transfer in pHsensitive hydrogels, Chem. Eng. Sci., 44 (1989) 559 566.

3 R.A. Siegel, M. Falamarzian, B.A. Firestone and B.C. Moxley, pH Controlled release from hydrophobic/po lyelectrolyte copolymer hydrogels, J. Controlled Release, 8 (1988) 179-182.

4 L. Brannon-Peppas and N.A. Peppas, Solute and penetrant diffusion in swellable polymers. IX. The mechanisms of drug release from $\mathrm{pH}$-sensitive swellingcontrolled systems, J. Controlled Release, 8 (1989) 267-274.

5 W.R. Good, Diffusion of water soluble drugs from initially dry hydrogels, in: R. Kostelnik (Ed.), Polymeric Delivery Systems, Gordon and Breach, New York, NY, 1976, pp. 139-156.

6 F.B. Rudolph, Diffusion in a multicomponent inhomogeneous multiphase system with moving boundaries. I. Swelling at constant volume, J. Polym. Sci., Polym. Phys. Ed., 17 (1979) 1709-1718.

7 F.B. Rudolph, Diffusion in a multicomponent inhomogeneous multiphase system with moving boundaries. II. Increasing or decreasing volume, J. Polym. Sci., Polym. Phys. Ed., 18 (1980) 2323-2336.

8 N.A. Peppas, R. Gurny, E. Doelker and P. Buri, Modelling of drug diffusion through swellable polymeric systems, J. Membrane Sci., 7 (1980) 241-253.

9 R.W. Korsmeyer, S.R. Lustig and N.A. Peppas, Solute and penetrant diffusion in swellable polymers. I. Mathematical modeling, J. Polym. Sci., Polym. Phys. Ed., 24 (1986) 395-408.

10 P.I. Lee, Diffusional release of a solute from a polymeric matrix - approximate analytical solutions, J. Membrane Sci., 7 (1980) 255-275.

11 J. Klier and N.A. Peppas, Solute and penetrans diffusion in swellable polymers. VIII. Influence of the swelling interface number on solute concentration profiles and release, J. Controlled Release, 7 (1988) 61-68.

12 H. Yasuda, C.E. Lamaze and L.D. Ikenberry, Permeability of solutes through hydrated polymer membranes, Makromol. Chem., 118 (1968) 19-35.

13 B. Carnahan, H.A. Luther and J.O. Wilkes, Applied Numerical Methods, Wiley, New York, NY, 1969, p. 74.

14 R.W. Korsmeyer, E. Von Meerwall and N.A. Peppas, Solute and penetrant diffusion in swellable polymers.
II. Verification of theoretical models, J. Polym. Sci., Polym. Phys. Ed., 24 (1986) 409-434.

15 J. Crank, Mathematics of Diffusion, Oxford University Press, Oxford, 1975, p. 47.

\section{APPENDIX}

\section{Model to analyze the effect of penetrant concentration gradient in a swelling gel matrix on drug release rate}

A one-dimensional model to describe drug release from a swelling matrix can be expressed by:

$\frac{\partial C_{2}}{\partial t}=\frac{\partial}{\partial x}\left[D_{2}\left(C_{1}\right) \frac{\partial C_{2}}{\partial x}\right]$

$\frac{\partial C_{1}}{\partial t}=\frac{\partial}{\partial x}\left(D_{1} \frac{\partial C_{1}}{\partial x}\right)$

and

$D_{2}=D_{2,0} \mathbf{e}^{-k_{f}(1 / H-1)}$

$D_{1}=$ constant

The subscripts 1 and 2 refer to penetrant and drug, respectively. The system is subject to the following boundary and initial conditions:

$C_{2}(L, t)=C_{2}(-L, t)=0$

$C_{1}(L, t)=C_{1}(-L, t)=C_{1, \infty}$

$C_{2}(x, 0)=C_{2,0}$

$C_{1}(x, 0)=C_{1,0}$

where $L$ is the half thickness of the slab, $C_{1,0}$ is the equilibrium penetrant concentration at time $0, C_{1, \infty}$ is the equilibrium penetrant concentration at time $\infty$, and $C_{2,0}$ is the initial drug loading concentration. In the exact treatment, $H$ can be expressed in terms of $C_{1}(x, t)$ by:

$H\left(C_{1}\right)=\frac{C_{1} L}{C_{1} L+M_{\mathrm{p}}}$

where $M_{\mathrm{p}}$ is the polymer weight in the gel sample and $L$ is the polymer thickness. Here we assume that cross sectional area is unity. The spatially averaged $H(t)$ can be expressed in 
terms of $M_{1}(t)$, the mass of penetrant sorbed in the matrix at an arbitrary time $t$, by

$H(t)=\frac{M_{1}(t)+M_{1,0}}{M_{1}(t)+M_{1,0}+M_{\mathrm{p}}}$

where $M_{1,0}$ is the initial weight of penetrant in the gel. Since $M_{1,0}=C_{1,0} L$ and $M_{1, \infty}=$ $\left(C_{1, \infty}-C_{1.0}\right) L$, it follows that:

$H(t)=\frac{\frac{M_{1}(t)}{M_{1, \infty}}+\frac{C_{1,0}}{C_{1, \infty}-C_{1,0}}}{\frac{M_{1}(t)}{M_{1, \infty}}+\frac{C_{1,0}}{C_{1, \infty}-C_{1,0}}+\frac{M_{\mathrm{p}}}{\left(C_{1, \infty}-C_{1,0}\right) L}}$

The solutions for $C_{1}(x, t)$ and $M_{1}(t) / M_{1, \infty}$ are readily available [15]. Therefore, the drug concentration profile can be solved by using eqns. (A1), (A3), (A5), and either (A7) or (A8). The fraction released can be evaluated simply by:

$\frac{M_{2}(t)}{M_{2, \infty}}=1-\frac{1}{2 C_{2,0} L} \int_{-L}^{L} C_{2}(x, t) \mathrm{d} x$

where $C_{2}$ is numerically solved via a finite difference scheme. The simulation assumed $M_{\mathrm{p}}=0.01$ and $L=1$; other parameters are specified in the text. $C_{1,0}$ and $C_{1, \infty}$ can be back calculated by assuming a value for $H(0)$ and $H(\infty)$. The final fraction released is plotted against a dimensionless time $\tau$ which is defined as $D_{2,0} t / L^{2}$. 